\title{
Anti-Malarial Targeting and Dosing Practices among Health Workers at Lower Level Health Facilities in Uganda
}

\author{
Stella Kakeeto ${ }^{1}$, Humphrey Wanzira ${ }^{2}$, Gerald Kagambirwe Karyeija ${ }^{3}$, Moses Kamya ${ }^{4}$, \\ Hasifa Bukirwa ${ }^{1}$ \\ ${ }^{1}$ School of Public Health, College of Health Sciences, Makerere University, Kampala, Uganda \\ ${ }^{2}$ African Field Epidemiology Network Fellow, National Malaria Control Program, Ministry of Health, \\ Kampala, Uganda \\ ${ }^{3}$ Department of Political and Administrative Sciences, Uganda Management Institute, Kampala, Uganda \\ ${ }^{4}$ Clinical Epidemiology Unit, College of Health Sciences, Makerere University, Kampala, Uganda \\ Email: "Kakeeto stella@yahoo.com, wanzirah@yahoo.com, gerald.karyeija@gmail.com, mka- \\ mya@infocom.co.ug, hbukirwa@hotmail.com
}

Received 6 July 2014; revised 21 August 2014; accepted 5 September 2014

Copyright (C) 2014 by authors and Scientific Research Publishing Inc.

This work is licensed under the Creative Commons Attribution International License (CC BY). http://creativecommons.org/licenses/by/4.0/

(c) () Open Access

\section{Abstract}

Background: Health worker shortages remain a significant challenge to delivery of health care services globally. Moving tasks, where appropriate, to less specialized health workers is recommended by the World Health Organization as a strategy to address this challenge. However, this concept is feared to raise specific quality concerns. This research aimed at assessing the performance of health workers to correctly prescribe (target) appropriate antimalarial treatment. Methods: We conducted a cross sectional study at three public health centre IVs in Uganda, with varying malaria transmission intensities (Kihihi-low, Kasambya-medium and Nagongera-high). We categorized prescribers into two groups: specialized prescribers (doctors and clinical officers) and less specialized prescribers (nurses and midwives). At each site, 100 records of patients seen between September and November 2011 and prescribed an antimalarial were retrieved for each group of prescribers. Correctness of the antimalarial drug prescribed and dose given were assessed for each group and compared to the 2005 Uganda national malaria treatment guidelines which recommend Artemether Lumefantrine (AL) for treatment of uncomplicated malaria and Quinine for complicated malaria. Results: Findings of the study showed that specialized health workers were more likely to target correctly as compared to the less specialized health workers [OR = 1.49 (1.00 - 2.22), $p=0.046]$. Appropriateness of dosing was higher among specialized prescribers compared to less specialized prescribers however this was not significant $[0 R=1.58$

${ }^{*}$ Corresponding author.

How to cite this paper: Kakeeto, S., Wanzira, H., Karyeija, G.K., Kamya, M. and Bukirwa, H. (2014) Anti-Malarial Targeting and Dosing Practices among Health Workers at Lower Level Health Facilities in Uganda. Health, 6, $2154-2161$.

http://dx.doi.org/10.4236/health.2014.616250 
(0.77 - 3.25), $p=0.206$ ]. Age of the participants, history of fever, diagnosis of malaria and prescription experience were not associated with correct targeting. Conclusion: This study shows that task shifting at the targeting level is not suitable; however, there is inadequate evidence to show that this also applies to anti-malarial dosing. Task shifting for the treatment of Malaria in Uganda should be investigated further using larger studies if it is to be considered as an option for solving the health worker shortages especially in regions with few specialized health workers but high malaria burden.

\section{Keywords}

Task Shifting, Anti-Malarial, Prescription, Dosing

\section{Background}

Acute health workforce shortages are a significant problem worldwide, with more than a billion people with little or no access to health services [1]. Uganda's health workforce shortages have worsened over the past decade; health worker levels are far below the WHO minimum threshold of 2.3 doctors, nurses and midwives per 1000 people [2]. The situation is even more desperate in rural areas where $80 \%-90 \%$ of the Uganda population lives, relying primarily on health services provided at low level health centres that are mainly managed by nurses.

Efforts to improve access to health care services have recently expanded to allow less qualified health workers participate in case management, an arrangement commonly known as task shifting [2]. The approach was found necessary to allow patients to have better access to health care services. However, it is feared to raise specific quality concerns including: incorrect diagnosis and treatment and inability to deal with complications, risk of decreased quality of patient care particularly if medical judgment and decision making is transferred.

There is contradicting evidence in the performance of health workers in targeting and dosing antimalarials. Ucakacon [3] found that doctors were more likely to conform to malaria treatment guidelines when compared to nurses. Only about 1 in 8 of the prescriptions by nurses conformed to the new antimalarial treatment policy. Similarly, USAID's ASSIST project reported that nurses and nursing assistants in Butebo HC IV who were prescribing antimalarials could not confidently rule out malaria in patients that tested negative in the lab [4].

Contrarily to this, Zurovac's study conducted at government health facilities in Kenya found that more specialised health workers made more errors: both major and minor errors in prescribing antimalarials to patients [5]. Another study comparing the performance of higher level health worker cadres to lower cadres found minimal differences in outcomes to patients [6].

This study was therefore conducted to compare the prescribing and dosing practices of specialised and less specialised health workers in three selected health centres in Uganda, as a means to evaluate whether task shifting is a viable option for the treatment of malaria. This study was nested in the Uganda Malaria Surveillance Project (UMSP) which collects accurate and timely data on malaria morbidity and mortality.

\section{Methods}

\subsection{Study Design and Setting}

This was a facility based cross sectional study conducted over a period of three months from September 2011 to November 2011. The study was conducted in three Health centres IVs located in areas with varying malaria transmission intensities of low (Kihihi) medium (Kasambya) and high (Nagongera) health centre IVs (Figure 1). The health centres studied are under a sentinel site malaria surveillance programme-the Uganda Malaria Surveillance Project (UMSP), established in six public health facilities in Uganda with support from the U.S President's Malaria Initiative (PMI). They are government run facilities, providing primarily out-patient services to the rural masses, with a catchment of about 100,000 people. The health centres receive an average of about 2000 patients every month, who are mainly managed by clinicians, nurses and midwives. More than $95 \%$ of the suspected malaria cases that present to these health centres have a malaria test done mostly by microscopy. Details of the sentinel sites have previously been described at length [7] [8]. 


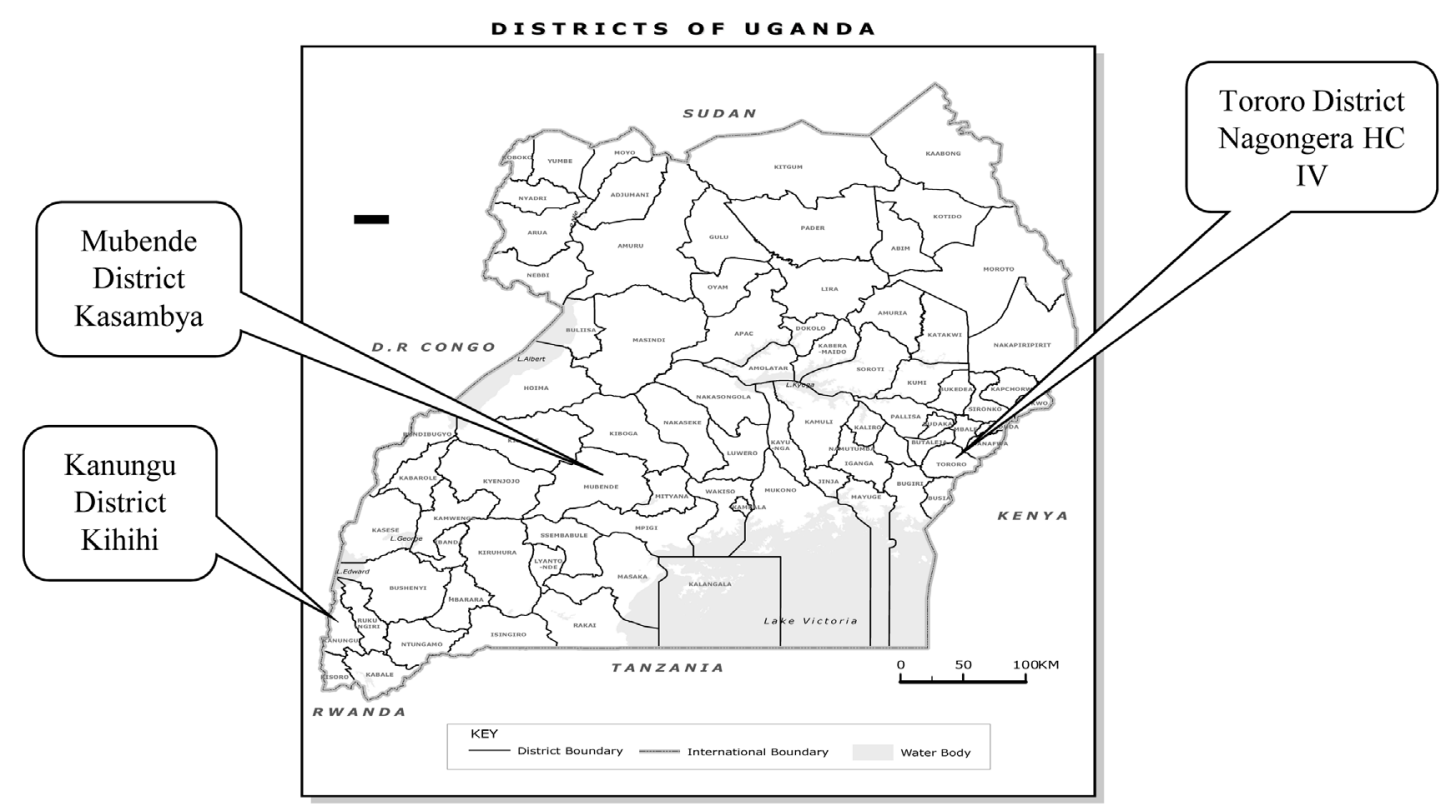

Figure 1. Map of the districts and health centres where the research was conducted.

\subsection{Study Procedures}

Individual level data of the selected case records was collected and reviewed. Each out-patient who presented to health centre was clerked using standardised case record form completed by a health worker during the patient encounter. The data that was collected included: patient demographics, malaria laboratory test results, diagnoses, medications prescribed and dispensed, and dosage for each medication.

A total of 19 health workers were involved in the study; the health workers selected are those who were involved in prescribing antimalarials to patients. Six were clinical officers that were categorised as the specialised health workers and 13 health workers (including one nursing officer, one comprehensive nurse, three registered nurses, three enrolled nurses, two registered midwives, one dental officer, one nursing assistant and one lab assistant) that were categorised as less specialised health workers. Health facility staff documents were reviewed in order to obtain health worker information.

\subsection{Sample Size and Study Variables}

All cases considered in this study were outpatients, with more than 95\% of them having a diagnosis of uncomplicated malaria. Using Kirkwood's [9] formulae for determining sample size of proportions, a total of 600 antimalarial prescriptions were reviewed to understand the appropriateness of anti-malarial regimen prescribed and the dosage given by the health workers. To achieve this, 200 patient charts were systematically randomly selected from each health centre, 100 charts for each category of prescribers. Only patient charts with documented age, weight and antimalarial prescription were included in the study. A prescription was considered accurately targeted if it had a positive blood smear for malaria and was given the appropriate antimalarial treatment according to the national antimalarial treatment guidelines [10]. Similarly, a prescription was considered correctly dosed if it was accurately targeted, with correctly documented number of drugs given, frequency and time the drugs should be taken as spelt out in the national treatment guidelines [10].

\subsection{Data Analysis}

Statistical analysis of the data was done using Stata 10. The analysis was done at two levels using mantel haeszel methods to estimate the odds ration and 95\% CF. In all analysis, a p-value of $<0.05$ was considered statistically significant. The first level of analysis assessed the association between health worker qualification and the following characteristics; Age, History of fever, correct diagnosis of malaria and correct dosing of antimalarials. The second level of analysis assessed whether targeting of antimalarials was affected by other factors 
associated with the health worker apart from the health worker qualification. These factors included; health worker category, years of experience, training and gender.

\subsection{Ethical Considerations}

The study was conducted using data collected under the Uganda Malaria Surveillance Project routine surveillance.

\section{Results}

\subsection{Health Worker Characteristics}

A total of 19 prescribers were included in the study from the health facilities.. Six were specialized prescribers (all clinical officers) and 13 less specialized prescribers (one nursing officer, one comprehensive nurse, three registered nurses, three enrolled nurses, two registered midwives, one dental officer, one nursing assistant and one lab assistant). Six prescribers were from Kihihi HC (one clinical officer, one nursing officer, one registered nurse, one enrolled nurse, one dental officer and one registered midwife). Kasambya HC had six health workers (two clinical officers, two enrolled nurses, one registered nurse and one lab assistant). The remaining 7 were from Nagongera HC (three clinical officers, one comprehensive nurse, one nursing assistant and one registered midwife). Nine prescribers were male (five specialized and four less specialized) and ten were female (one specialized and nine less specialized). 15 prescribers had more than two years of work experience in prescribing and dosing antimalarials. Only four prescribers had less than two years of experience in malaria case management, three of whom were less specialized.

\subsection{Prescription/Study Participant Characteristics}

A total of 600 antimalarial prescriptions made by the 19 prescribers were reviewed. From each health centre, 200 prescriptions were reviewed; 100 for the specialised health workers and 100 for the less specialised health workers. This totalled to 300 prescriptions for the specialised health workers and 300 for the less specialised health workers. $250(41.7 \%)$ prescriptions belonged to patients $\leq 5$ years of age and $350(58.3 \%)$ prescriptions to patients $>5$ years of age. More than half of the prescriptions belonged to male health workers $(\mathrm{n}=369,61.5 \%)$. Almost all the patients had their weight recorded $(n=586,97.7 \%) 562(94.5 \%)$ patient prescriptions had a laboratory test for malaria done with microscopy. Similarly, 521 (92.5\%) patients were examined for history of fever (Table 1).

\subsection{Association of Selected Indicators with Health Worker Category}

Age of participants was equally distributed between the specialised and less specialised health workers. Specialised health workers were $21 \%$ more likely to examine for history of fever, $58 \%$ more likely to correctly diagnose malaria, and $27 \%$ more likely to give the correct dosage. However, all these were not statistically significant as seen from Table 2.

\subsection{Health Worker Factors that Affect Correct Targeting}

When health worker factors were considered for correct targeting, we found out that specialized health workers were $49 \%$ more likely to target correctly as compared to the less specialized health workers, and this was significant (OR $=1.49,95 \% \mathrm{CI} 1.00-2.22)$. We further found that if a health worker was specialized, had more than two years of work experience, had received training in malaria case management and was male, they were more likely to target antimalarials correctly. However, these results were not significant as seen from Table 3 .

\section{Discussion}

This study showed that specialized health workers were significantly more likely to correctly diagnose malaria and give the correct antimalarial dose to a patient as compared to the less specialized health workers. When health worker factors were considered for correct targeting, still, the specialized health workers were more likely to target correctly as compared to the less specialized health workers, although this was not significant, probably 
Table 1. Distribution of Study participants’ baseline characteristics.

\begin{tabular}{|c|c|c|}
\hline \multirow{2}{*}{ Characteristic } & \multicolumn{2}{|c|}{ Study participants $N=600$} \\
\hline & Number & Percentage \\
\hline $\begin{array}{c}\text { Site } \\
\text { Kasambya HC IV } \\
\text { Kihihi HC IV } \\
\text { Nagongera HC IV }\end{array}$ & $\begin{array}{l}200 \\
200 \\
200\end{array}$ & $\begin{array}{l}33.33 \\
33.33 \\
33.33\end{array}$ \\
\hline $\begin{array}{l}\text { Age category in years } \\
\leq 5 \\
>5\end{array}$ & $\begin{array}{l}250 \\
350\end{array}$ & $\begin{array}{l}41.67 \\
58.33\end{array}$ \\
\hline $\begin{array}{c}\text { Gender } \\
\text { Female } \\
\text { Male }\end{array}$ & $\begin{array}{l}231 \\
369\end{array}$ & $\begin{array}{l}38.50 \\
61.50\end{array}$ \\
\hline $\begin{array}{l}\text { Recorded weight } \\
\text { Recorded } \\
\text { Not recorded }\end{array}$ & $\begin{array}{c}586 \\
14\end{array}$ & $\begin{array}{c}97.67 \\
2.33\end{array}$ \\
\hline $\begin{array}{l}\text { History of fever } \\
\text { Yes } \\
\text { No }\end{array}$ & $\begin{array}{c}521 \\
42\end{array}$ & $\begin{array}{c}92.54 \\
7.46\end{array}$ \\
\hline $\begin{array}{l}\text { BS for Malaria }{ }^{\#} \\
\text { BS done } \\
\text { BS not done }\end{array}$ & $\begin{array}{c}562 \\
33\end{array}$ & $\begin{array}{c}94.45 \\
5.55\end{array}$ \\
\hline $\begin{array}{c}\text { Prescribed anti-malarials } \\
\text { Coartem } \\
\text { Quinine } \\
\text { Artesunate } \\
\text { Artemether Injection } \\
\text { Arco } \\
\text { Coartem and Quinine }\end{array}$ & $\begin{array}{c}514 \\
76 \\
4 \\
1 \\
1 \\
4\end{array}$ & $\begin{array}{c}85.67 \\
12.67 \\
0.67 \\
0.17 \\
0.17 \\
0.67\end{array}$ \\
\hline
\end{tabular}

*37 missing history of fever record; ${ }^{*} 5$ missing BS for Malaria.

Table 2. Association of selected indicators with health worker category.

\begin{tabular}{|c|c|c|c|c|}
\hline \multirow{3}{*}{ Indicator } & \multicolumn{2}{|c|}{ Health Worker category } & \multirow[b]{3}{*}{ OR $(95 \% \mathrm{CI})$} & \multirow[b]{3}{*}{ p-value } \\
\hline & Specialized & Less specialized & & \\
\hline & N (\%) & N (\%) & & \\
\hline \multicolumn{5}{|c|}{ Age category in years } \\
\hline$\leq 5$ & $125(41.67)$ & 125 (41.67) & & \\
\hline$>5$ & $175(58.33)$ & $175(58.33)$ & $1.00(0.72-1.38)$ & 1.000 \\
\hline \multicolumn{5}{|c|}{ Examined for history of fever } \\
\hline Yes & 260 (93.19) & $261(91.90)$ & & \\
\hline No & $19(6.81)$ & $23(8.10)$ & $1.21(0.64-2.27)$ & 0.561 \\
\hline \multicolumn{5}{|c|}{ Correctly diagnosed with malaria } \\
\hline Yes & $285(95.64)$ & $277(93.27)$ & & \\
\hline No & $13(4.36)$ & $20(6.73)$ & $1.58(0.77-3.25)$ & 0.206 \\
\hline \multicolumn{5}{|c|}{ Correctly written prescription dosages } \\
\hline Yes & $236(78.67)$ & $223(74.33)$ & & \\
\hline No & $64(21.33)$ & $77(25.67)$ & $1.27(0.87-1.86)$ & 0.211 \\
\hline
\end{tabular}

due to the small sample size of health workers. This could have resulted into selection bias since the same prescribers treated the study participants. If a health worker was specialized, had more than two years of work experience, had received training in malaria case management and was male, they were more likely to target antimalarials correctly.

The prescription pattern found in this study shows that most specialised health workers were in position to correctly prescribe/target antimalarials as compared to the less specialised health workers. These findings concur with Ucakacon's [3] study where doctors and clinical officers in Uganda were more likely to conform to the 
Table 3. Health worker factors that affect correct targeting.

\begin{tabular}{|c|c|c|c|c|}
\hline \multirow{3}{*}{ Indicator } & \multicolumn{2}{|c|}{ Targeting } & \multirow[b]{3}{*}{ OR (95\% CI) } & \multirow[b]{3}{*}{ p-value } \\
\hline & Correct & Incorrect & & \\
\hline & N (\%) & $\mathbf{N}(\%)$ & & \\
\hline \multicolumn{5}{|c|}{ Health worker category } \\
\hline Specialized & $246(52.12)$ & 54 (42.19) & & \\
\hline Less specialized & $226(47.88)$ & $74(57.81)$ & $1.49(1.00-2.22)$ & 0.046 \\
\hline \multicolumn{5}{|l|}{ Years of experience } \\
\hline$\leq 2$ & 8 (1.69) & $5(3.91)$ & & \\
\hline$>2$ & $464(98.31)$ & 123 (96.09) & $0.42(0.14-1.32)$ & 0.128 \\
\hline \multicolumn{5}{|c|}{ Training in Malaria case management } \\
\hline No & $27(5.72)$ & $10(7.81)$ & & \\
\hline Yes & 445 (94.28) & 118 (92.19) & $0.72(0.34-1.52)$ & 0.383 \\
\hline \multicolumn{5}{|c|}{ Gender of health worker } \\
\hline Female & $139(29.45)$ & 47 (36.72) & & \\
\hline Male & $333(70.55)$ & $81(63.28)$ & $0.72(0.48-1.09)$ & 0.115 \\
\hline
\end{tabular}

antimalarial treatment guidelines as compared to the nurses. Zurovac [11] also found that higher level health worker cadres had better prescribing practices and Ameme's [12] study in Ghana found conformity to treatment guidelines higher in hospitals than lower health facilities that are mostly staffed with lower cadre health workers. Potential explanation for this difference could be that the specialized health workers are formally trained to prescribe whereas the nurses only prescribe due to shortage of clinicians but this is not primarily their role. This finding is worrying because majority of the health facilities in Uganda especially the low level facilities are managed by less specialised health workers.

Some studies have however found less specialised health workers with higher odds of correctly prescribing/targeting antimalarials. Zurovac [5] in Kenya found that nursing aides who were considered less trained complied much more closely to treatment guidelines than clinical officers. They were also found better at making consultations and following recommended guidelines than medical officers.

Inspite of the better performance of specialised health workers at prescribing antimalarials as compared to the less specialised health workers, the rate of appropriate use of the antimalarials was generally impressive. Majority of the patients with uncomplicated malaria were prescribed an Artemesinin based Combination Therapy (ACT), precisely Artemether Lumefantrine (AL), which is the recommended first line therapy for uncomplicated malaria in Uganda [10]. This is an encouraging finding given the high burden of malaria in many parts of the country and the efficacy of ACTs for uncomplicated malaria [13]-[16].

There was no significant difference between specialised and less specialised health workers in dosing antimalarials. Specialised health workers were $27 \%$ more likely to dose appropriately as compared to the less specialised health workers with about $25 \%$ of the dosages given by less specialised health workers as incorrect. This could possibly be that less specialised health workers are not formally trained to prescribe and dose medicines especially the nursing aids as it is for the specialised health workers These findings are consistent with the results of two studies where most of the inappropriate dosing seen among the less specialised health workers happened in lower age groups. A study done in Tanzania [17] found incorrect dosing of AL among children aged 3 12 years in addition to another study [5] in Kenya which found more appropriate treatment among infants than older children.

Health workers that had more than two years of work experience were more likely to prescribe/target antimalarials correctly. This is supported by the fact that health workers that have spent more years in practice may have learnt through experience the appropriate care for patients, are more likely to have received training in malaria case management and might have had several mentorship opportunities in the course of their practice, building confidence in prescribing antimalarials [18]. However, there is one study done in Uganda that was contrary to what was found in this study. Ucakacon [3] found that health workers that had been in service for a shorter period were more likely to conform to the antimalarial treatment policy as compared to those that had been in service longer. This finding could have been due to the time of study conduction when training following new guidelines was in its early phases and therefore, there is a possibility that the health workers that had been in service longer had not adapted to the new guidelines yet. 


\section{Conclusion}

The findings from this study showed that task shifting at the targeting level is not suitable. However, there is inadequate evidence to show that this also applies to dosing. These findings may not be generalised since the health facilities considered were few and under a rigorous malaria surveillance project. For task shifting to be considered as an option, we therefore recommend larger studies to evaluate its viability especially in areas with few specialised health workers but high in malaria.

\section{Contributions}

SK and HB conceived, designed, and conducted this study. HW supported the data analysis. SK took lead in writing the manuscript and the other authors (HW, AK and $\mathrm{MK}$ ) contributed towards writing the manuscript.

\section{Acknowledgements}

We appreciate the staff and administration of Kihihi, Kasambya and Nagongera Health centres for their support and cooperation during the study period, and thank all members of the study team for their commitment and dedication to getting this work done in time. We would also like to thank the Uganda Malaria Clinical Operational and Health Services (COHRE) Training Program at Makerere University, Grant \#D43-TW00807701A1, from the Fogarty International Centre (FIC) at the National Institutes of Health (NIH) that financially supported this research.

\section{References}

[1] WHO (2006) The World Health Report: Working Together for Health. World Health Organisation, France.

[2] WHO (2007) Strengthening Health Services to fight HIV/AIDs. World Health Organisation, Geneva.

[3] Ucakacon, P.S., Achan, J., Kutyabam, P., Odoi, A.R. and Kalyango, N.J. (2011) Prescribing Practices for Malaria in a Rural Ugandan Hospital: Evaluation of a New Malaria Treatment Policy. African Health Sciences, 11, S53-S59.

[4] Rotich, L. (2011) Reducing Prescription of Antimalarials to Smear Negative Patients. USAID ASSIST Project, April 13, Kampala.

[5] Zurovac, D., Rowe, A.K., Ochola, S.A., Noor, A.M., Midia, B., English, M., et al. (2004) Predictors of the Quality of Health Worker Treatment Practices for Uncomplicated Malaria at Government Health Facilities in Kenya. International Journal of Epidemiology, 33, 1080-1091. http://dx.doi.org/10.1093/ije/dyh253

[6] Delanyo, D. (2004) Using Mid-Level Cadres as Substitutes for Internationally Mobile Health Professionals in Africa. A Desk Review. Human Resources for Health, 2, 7. http://dx.doi.org/10.1186/1478-4491-2-7

[7] Sserwanga, A., Harris, J.C., Kigozi, R., Menon, M., Bukirwa, H., Gasasira, A., Kakeeto, S., Kizito, F., Quinto, E., Rubahika, D., Nasr, S., Filler, S., Kamya, M.R. and Dorsey, G. (2011) Improved Malaria Case Management through the Implementation of a Health Facility Sentinel Site Surveillance System in Uganda. PLoS One, 6, e16316.

[8] Kigozi, R., Baxi, S.M., Gasasira, A., Sserwanga, A., Kakeeto, S., Nasr, S., Rubahika, D., Dissanayake, G., Kamya, M.R., Filler, S. and Dorsey, G. (2012) Indoor Residual Spraying of Insecticide and Malaria Morbidity in a High Transmission Intensity Area of Uganda. PLoS One, 7, e42857.

[9] Kirkwood, B. and Sterne, J.A.C. (2001) Essential Medical Statistics. 2nd Edition, Blackwell Publishing, Oxford, 35.

[10] MoH (2005) National Policy on Malaria Treatment. In: National Malaria Control Program MoHU, Editor. Kampala.

[11] Zurovac, D., Njogu, J., Akhwale, W.S., Hamer, D.H. and Snow, R.W. (2008) Translation of Artemether-Lumefantrine Treatment Policy into Paediatric Clinical Practice: An Early Experience from Kenya. Tropical Medicine \& International Health, 13, 99-107. http://dx.doi.org/10.1111/j.1365-3156.2007.01980.x

[12] Ameme, D.K., Nyarko, K.M., Malm, K.L., Afari, E.A., Wurapa, F. and Sackey, S. (2012) Prescribing Practices for Uncomplicated Malaria in a Rural District in Ghana. Internation Journal of Tropical Diseases, 4.

[13] Dorsey, G., Staedke, S., Clark, T.D., Njama-Meya, D., Nzarubara, B., Maiteki-Sebuguzi, C., Dokomajilar, C., Kamya, M.R. and Rosenthal, P.J. (2007) Combination Therapy for Uncomplicated Falciparum Malaria in Ugandan Children. JAMA, 297, 2210-2219. http://dx.doi.org/10.1001/jama.297.20.2210

[14] Kamya, M.R., Yeka, A., Bukirwa, H., Lugemwa, M., Rwakimari, J.B., Staedke, S.G., Talisuna, A.O., Greenhouse, B., Nosten, F., Rosenthal, P.J., et al. (2007) Artemether-Lumefarntrine versus Dihydroartemesinin-Piperaquine for Treatment of Malaria: A Randomised Trial. PLOS Clinical Trials, 2, e20.

[15] Yeka, A., Dorsey, G., Kamya, M.R., Talisuna, A., Lugemwa, M., Rwakimari, J.B., Staedke, S.G., Rosenthal, P.J., 
Wabwire-Mangen, F. and Bukirwa, H. (2008) Artemether-Lumefantrine versus Dihydroartemesinin Piperaquine for Treating Uncomplicated Malaria: A Randomised Trial to Guide Policy in Uganda. PLoS One, 3, e2390.

[16] Achan, J., Tibenderana, J.K., Kyabayinze, D., Mangen, F.W., Kamya, M.R., Dorsey, G., D’Alessandro, U., Rosenthal, P.J. and Talisuna, A.O. (2009) Effectiveness of Quinine versus Artemether-Lumefantrine for Treating Uncomplicated Falciparum Malaria in Ugandan Children: Randomised Trial. BMJ, 339, Article ID: b2763.

[17] Masanja, I.M., Selemani, M, Khatib, R.A., Amuri, B., Kuepfer, I., Kajungu, D., Savigny, D., Kachur, S.P. and Skarbinski, J. (2013) Correct Dosing of Artemether-Lumefantrine for Management of Uncomplicated Malaria in Rural Tanzania: Do Facility and Patient Characteristics Matter. Malaria Journal, 12, 446.

[18] Selemani, M., Masanja, I.M., Kajungu, D., Amuri, M., Njozi, M., Khatib, R.A., et al. (2013) Health Worker Factors Associated with Prescribing of Artemisinin Combination Therapy for Uncomplicated Malaria in Rural Tanzania. Malaria Journal, 12, 334. http://dx.doi.org/10.1186/1475-2875-12-334 
Scientific Research Publishing (SCIRP) is one of the largest Open Access journal publishers. It is currently publishing more than 200 open access, online, peer-reviewed journals covering a wide range of academic disciplines. SCIRP serves the worldwide academic communities and contributes to the progress and application of science with its publication.

Other selected journals from SCIRP are listed as below. Submit your manuscript to us via either submit@scirp.org or Online Submission Portal.
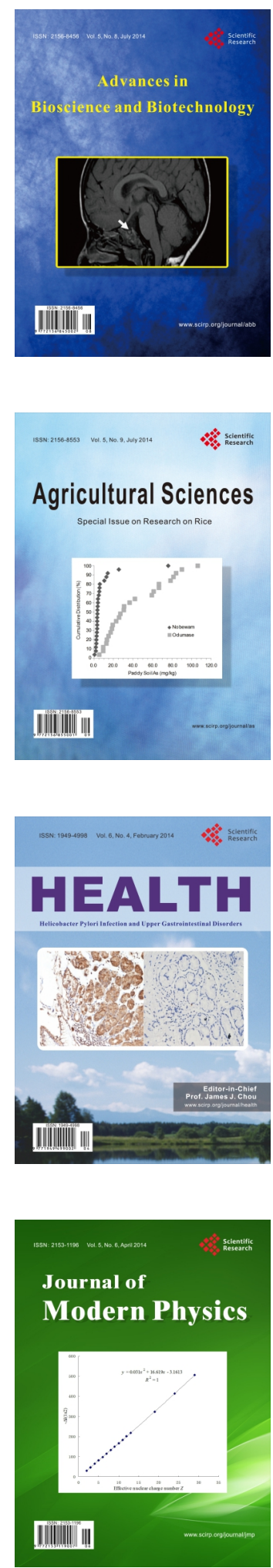
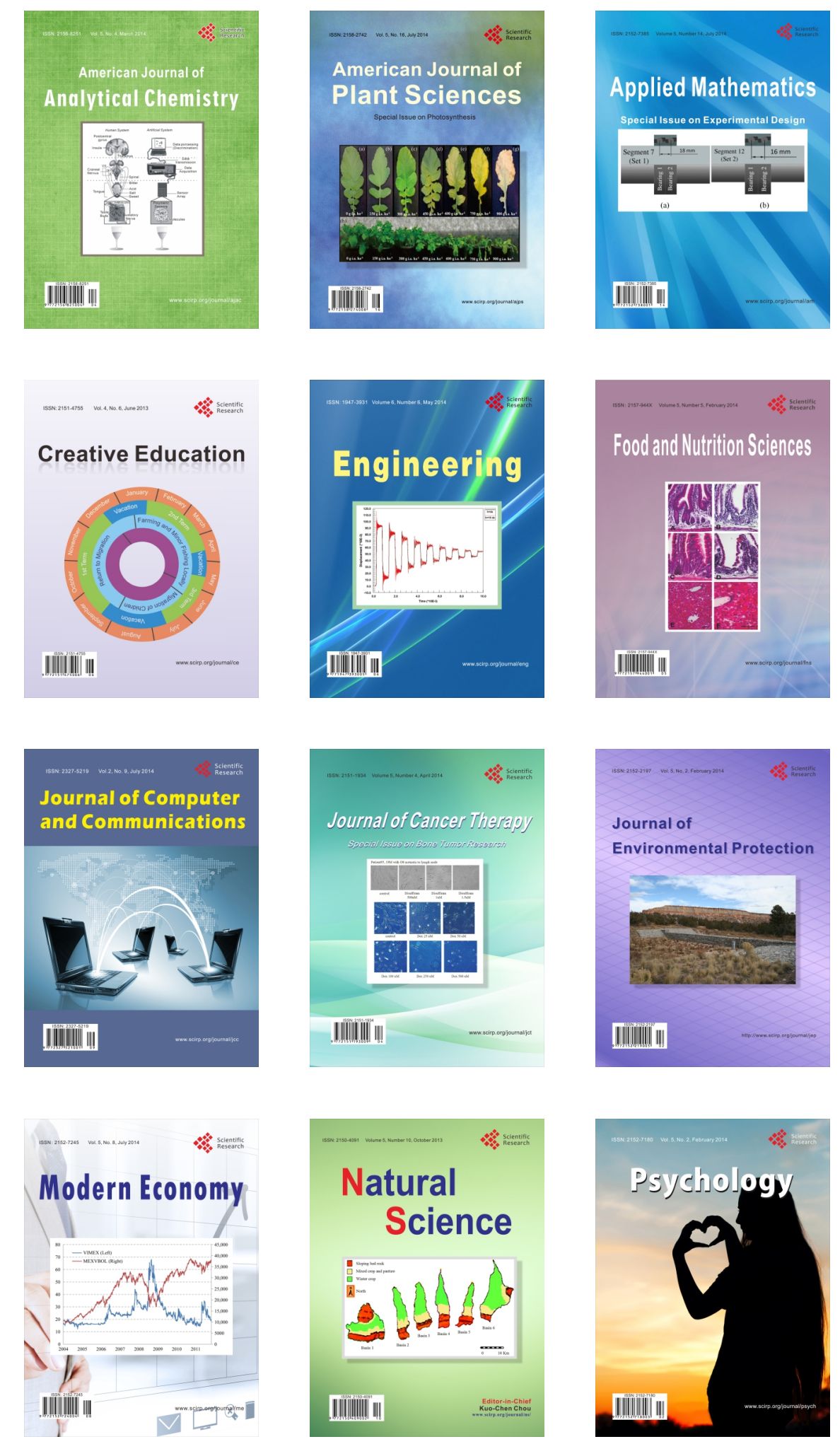